\title{
Generation of Four-Wave Mixing in a Highly Non-Linear Optical Fiber Using a Tunable Dual Wavelength Fiber Laser Source
}

\author{
Noor Ummi Hazirah Hani Zalkepali @ Zulkefli, Noor Azura Awang*, \\ Fatin Shaqira Abdul Hadi, Atiqah Nabiha Azmi and Zahariah Zakaria \\ Department of Physics and Chemistry, Faculty of Applied Sciences and Technology, UTHM Pagoh \\ Educational Hub, Universiti Tun Hussein Onn Malaysia, 84000, Pagoh, Johor, Malaysia.
}

Received 30 September 2017; accepted 29 June 2018; available online 1 August 2018

DOI: https://10.30880/jst.2018.10.02.028

\begin{abstract}
In this paper, we demonstrate a Four Wave Mixing (FWM) effect in a highly nonlinear single mode optical fiber. A tunable dual-wavelength fiber laser is used to provide the pump and signal probe to generate partially degenerate FWM. Calculations using coupled differential equations and experimental results in the generation of FWM were analyzed. We observed that FWM conversion efficiency depends more significantly on the wavelength detuning between pump and signal wavelength as compared to zerodispersion wavelength. The tunability is achieved using an arrayed waveguide grating which provides different wavelength combinations of the dual-wavelength fiber laser output and can be tuned from $1530.47 \mathrm{~nm}$ to $1548.61 \mathrm{~nm}$ with a spacing of $100 \mathrm{GHz}$. The highly nonlinear fiber has a nonlinear coefficient, $\gamma$ of $10.8 \mathrm{~W}^{-1} \mathrm{~km}^{-1}$. Hence, to achieve higher FWM output power the pump power of the dual wavelength fiber laser has to be higher than that of the signal probe. The optimum measured values of pump and signal power are $+13.3 \mathrm{dBm}$ and $+5 \mathrm{dBm}$ respectively, limited buy the current experimental setup.
\end{abstract}

Keywords: Four Wave Mixing (FWM); dual-wavelength fiber laser; Highly Nonlinear Fiber (HNLF); FWM conversion efficiency.

\section{Introduction}

Nonlinear optical phenomenon has become an important technique in the generation of new frequencies which finds many applications in the area of parametric oscillation, multiwavelength fiber laser, and wavelength conversion. Lately, the interest in all optical wavelength converter is to create a new platform for an all optical network system that will improve the network accessibility and flexibility, and to re-route the data traffic without having to convert them into electrical format [1-3].

Wavelength converter is used to convert one or more of the incoming wavelengths from a WDM system into a desired wavelength or group of wavelengths without significant distortion in the output signals and hence facilitate wavelengths reuse in the optical network. Various techniques for optical wavelength conversion have been reported which include the use of the nonlinear behavior in a Semiconductor Optical Amplifier (SOA) and in highly nonlinear optical fiber (HNLF). Generally, wavelength conversion can be achieved by using crossphase modulation (XPM) [4,5], cross gain modulation (XGM) [6,7] and through four wave mixing (FWM) [8-11]. Among these, four-wave mixing provides an interesting approach due to its simplicity. Although FWM can be observed in nonlinear medium such as SOA, but more interests are geared towards single mode optical fiber with zero dispersion at $1530 \mathrm{~nm}$ region, which provides an inexpensive medium and also the possibility of lower insertion loss in an actual system.

Generally, the fourth wavelength is generated from three different wavelengths input in a FWM phenomenon. The efficiency of this nonlinear interaction depends on the nonlinear phase mismatch and chromatic dispersion is a key parameter that determines this. In a special case like partial degeneration, only two wavelengths inputs are needed to generate the third wavelength. Hence, dualwavelengths fiber laser will be an interesting source to be used for FWM in a nonlinear medium. This has been demonstrated for the case of an SOA acting as the nonlinear medium $[12,13]$. 
In this paper, we provide a first time investigation on the FWM effect on a highly nonlinear fiber using a dual-wave fiber laser constructed in our laboratory as the dual input wavelength source (pump and probe signal) to generate the third wavelength. The usage of tunable dual-wavelength fiber laser is due to the fact that it is more effective compare to using two independent tunable laser sources as the pump and signal beams. It is known that zero-dispersion wavelength contributes to the high FWM conversion efficiency. However, in this experiment it is observed that the wavelength detuning of pump and signal wavelength has a more significant effect on the conversion efficiency of third wavelength. This demonstration provides results that indicate the insufficiency of the existing theoretical model to predict the power output of the newly generated FWM signals.

\section{Theoretical Background}

The generation of FWM in highly nonlinear fiber can be explained by using the coupled differential equations for the propagating amplitudes, including the contributions to phase mismatch due to XPM and SPM [14]. A well-known formula used for FWM estimation was originally derived by Hill et. al. [15] and reformulated later to include the phase mismatch dependent efficiency by Shibata et. al. [16]. In the FWM process, two chosen wavelengths ( $\lambda_{\text {pump }}$ and $\left.\lambda_{\text {signal }}\right)$ will generate a converted wavelength $\left(\lambda_{\text {converted signal }}=2 \lambda_{\text {pump }}-\lambda_{\text {signal }}\right)$ as shown in Fig. 1. For analyzing the efficiency of FWM generation, the phase mismatching condition has to be satisfied [17]. The efficiency of generating a converted wavelength in FWM process depends on the pump power, wavelength detuning, chromatic dispersion of the fiber and the fiber length. The phase mismatch, $\Delta k$, is the core parameter in determining the conversion efficiency, $\eta$, of the FWM. Basically, there are two types of phase mismatch that need to be considered, namely, the dispersive phase mismatch, $\Delta k_{l}$, and the nonlinear phase mismatch, $\Delta k_{n l}$, as shown in equation $1[14,16-20]$;

$$
\Delta k=\Delta k_{1}+\Delta k_{n}
$$

The dispersive phase mismatch can be calculated as;

$$
\begin{aligned}
& \Delta k_{1}=2 \pi \lambda_{o}^{2} c\left(\frac{1}{\lambda_{o}}-\frac{1}{\lambda_{p}}\right)\left(\frac{1}{\lambda_{p}}-\frac{1}{\lambda_{s}}\right) \\
& {\left[D_{c}+\frac{\lambda_{o}^{2}}{2}\left\{\left(\frac{1}{\lambda_{o}}-\frac{1}{\lambda_{p}}\right)+\left(\frac{1}{\lambda_{p}}-\frac{1}{\lambda_{s}}\right)\right\} \frac{d D_{c}}{d \lambda}\right]}
\end{aligned}
$$

where $\lambda_{o}$ is the zero-dispersion wavelength, $c$ is the speed of light, $\lambda_{p}$ is the pump wavelength, $\lambda_{s}$ is the signal wavelength, $D_{c}$ is the fiber dispersion as a function of $\lambda$, and $d D_{c} / d \lambda$ is the dispersion slope.

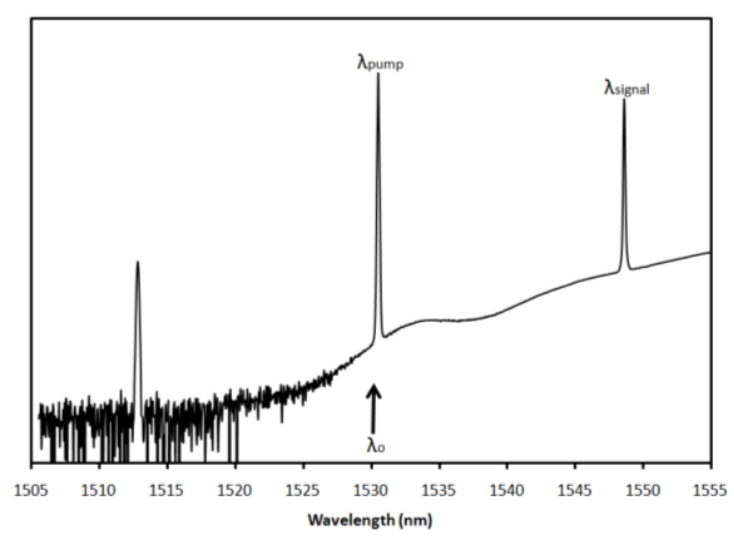

Fig. 1 The FWM effect in tunable dualwavelength fiber laser.

The nonlinear contribution to phase mismatch is analytically derived as,

$$
\Delta k_{n l}=\gamma\left(P_{p}-P_{s}\right)\left(\frac{1-e^{-\alpha L_{e f f}}}{\alpha L_{e f f}}\right)
$$

where $P_{P}$ is the pump power, $P_{S}$ is the signal power, $L_{\text {eff }}$ is the effective length of the fiber, defined in terms of the total fiber length $(L)$ and fiber attenuation $(\alpha)$ as,

$$
L_{e f f}=\frac{1-e^{-\alpha L}}{\alpha}
$$

and $\gamma$ is the nonlinear coefficient given by,

$$
\gamma=\frac{2 \pi n_{2}}{\lambda A_{\text {eff }}}
$$

where $A_{\text {eff }}$ is the effective fiber core area, $\lambda$ is the vacuum wavelength and $n_{2}$ is the nonlinear index coefficient of the fiber. After calculating 
the total phase mismatching, we can obtain FWM conversion efficiency which is defined as,

$$
\eta=\frac{\alpha^{2}}{\alpha^{2}+\Delta k^{2}}\left(1+\frac{2 e^{-\alpha L}(1-\cos (\Delta \kappa L))}{\left(1-e^{-\alpha L}\right)^{2}}\right)
$$

and FWM product power as given by,

$$
P_{F W M}=\frac{4 \eta}{9} \gamma^{2} P_{p} P_{s} e^{-\alpha L} L_{e f f}^{2}
$$

Theoretically, the FWM conversion efficiency is optimum when the phase mismatch condition is fulfilled. Phase mismatch condition can only fulfilled when the pump and signal interact at zero-dispersion wavelength and the wavelength detuning between pump and signal wavelengths is approaching one another. However, this might not be the case in actual experiment due to the gain-dependence of pump and signal that originates from the amplified spontaneous emission (ASE) output profile which is higher at $1560 \mathrm{~nm}$ that shown in Fig. 2. In this case, pump and signal wavelengths are situated closer to the higher ASE region will lead to higher FWM conversion efficiency compared to the one at the $1530 \mathrm{~nm}$ zero-dispersion.

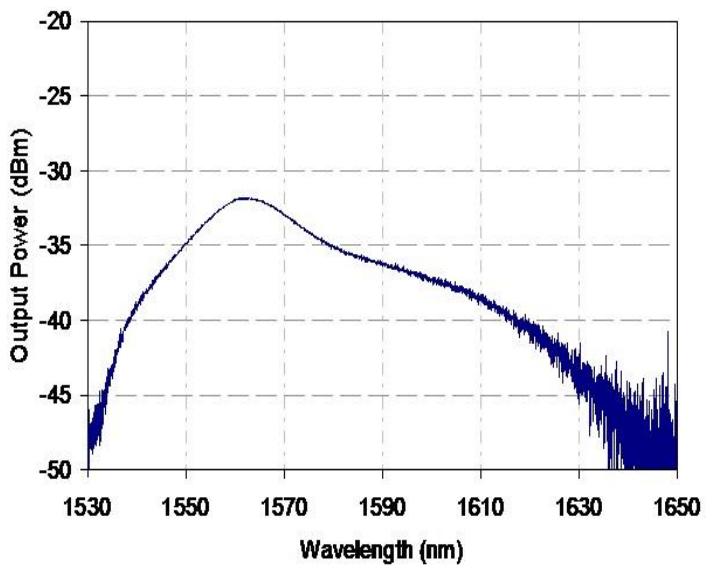

Fig. 2 The ASE spectrum of the $11 \mathrm{~m}$ metrogain EDF.

\section{Experimental Setup}

Fig. 3 shows the proposed ring cavity experimental setup which uses $11 \mathrm{~m}$ of Metrogainerbium doped fiber from Fibercore as an ASE source as well as the gain medium. The EDF with Erbium ion concentration of 900ppmwt has an absorption coefficient of $18.06 \mathrm{~dB} / \mathrm{m}$ and $11.3 \mathrm{~dB} / \mathrm{m}$ at $1530 \mathrm{~nm}$ and 980 $\mathrm{nm}$ respectively.

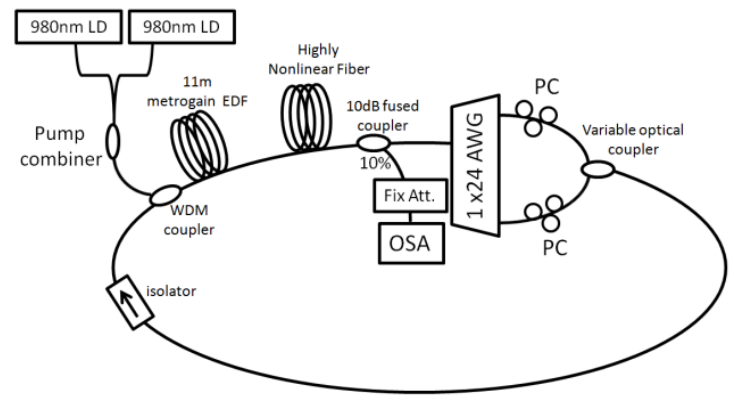

Fig. 3 Schematic diagram for generating FWM effect in a Highly Nonlinear Fiber using dual wavelengths fiber laser incorporating AWG as a tuning element.

In this experimental setup, we demonstrate a high-power erbium-doped fiber amplifier (EDFA) pumped with 980nm wavelength light from two semiconductor laser diodes. Light outputs from the two $980 \mathrm{~nm}$ pump LD modules are $215 \mathrm{~mW}$ each. They are combined using a polarization beam combiner (PBC). The output is then connected to the ring cavity via a $980 \mathrm{~nm} / 1550 \mathrm{~nm}$ wavelength division multiplexer (WDM) coupler and directed into the ring cavity. The combined pump power that travelled in clockwise direction is then absorbed by the EDF. The emission of C-band ASE output from the EDF will travel through a $1 \times 24$ Arrayed Waveguide (AWG) and was sliced into 24 individual output wavelength from each channel output. The 1x24 channels output cover the wavelength range from $1530.473 \mathrm{~nm}$ to $1548.613 \mathrm{~nm}$ with an interchannel spacing of $100 \mathrm{GHz}$. Two selected channels from the AWG outputs are connected to the polarization controllers for output optimization before being connected to a $2 \mathrm{x} 1$ variable optical coupler. Lastly, the variable optical coupler was connected to an optical isolator to complete the ring cavity which allows the light to propagate in single direction only. In the AWG channels, the signal wavelength is fixed at $1548.6 \mathrm{~nm}$ (channel 24) and the pump wavelength is varied from $1530.5 \mathrm{~nm}$ (channel 1) to $1545.4 \mathrm{~nm}$ (channel 23). The chosen channels are then connected to the variable optical coupler via a PC (polarization controller) as illustrated in Fig. 3. $\mathrm{PC}$ is used to maximize the matching of the 
polarization state of pump and signal power. The dual-wavelengths fiber laser is generated at the output port of the variable optical coupler and travels in the clockwise direction and will be amplified again by the gain medium. The high output power from the dual wavelengths fiber laser will travel through the $100 \mathrm{~m}$ highly nonlinear fiber from OFS which acts as the non-linear medium to generate the FWM output wavelength. This specification of highly nonlinear fiber is given in Table 1.

Finally, the output end of the HNLF is connected to a 90/10 coupler with the $10 \%$ port connected to an optical spectrum analyzer (Yokogawa aq6370B) via a $25 \mathrm{~dB}$ fix attenuator and the $90 \%$ port completes the loop by connecting to the AWG.

Table. 1 Fiber Parameters and Properties of Highly Nonlinear Fiber

\begin{tabular}{|c|c|c|}
\hline Length, $L$ & $\mathrm{~m}$ & 100 \\
\hline $\begin{array}{c}\text { Dispersion @ } \\
\text { 1550nm, } D_{c}\end{array}$ & $\mathrm{ps} / \mathrm{nm} \cdot \mathrm{km}$ & 0.15 \\
\hline $\begin{array}{c}\text { Dispersion slope @ } \\
1550 \mathrm{~nm}, d D_{c} / d \lambda\end{array}$ & $\mathrm{ps} / \mathrm{nm}^{2} \cdot \mathrm{km}$ & 0.007 \\
\hline $\begin{array}{c}\text { Zero dispersion } \\
\text { wavelength, } \lambda_{o}\end{array}$ & $\mathrm{~nm}$ & 1531 \\
\hline Cut off wavelength & $\mathrm{nm}$ & 1240 \\
\hline $\begin{array}{c}\text { Effective area @ } \\
1550 \mathrm{~nm}, A_{\text {eff }}\end{array}$ & $\mu \mathrm{m}$ & 12.3 \\
\hline $\begin{array}{c}\text { Nonlinear coefficient } \\
\text { estimated from } \\
\text { effective area, } \gamma\end{array}$ & $(\mathrm{W} . \mathrm{km})^{-1}$ & 10.8 \\
\hline $\begin{array}{c}\text { Fiber attenuation @ } \\
1550 \mathrm{~nm}, \alpha\end{array}$ & $\mathrm{dB} / \mathrm{km}$ & 0.73 \\
\hline $\begin{array}{c}\text { Total module loss @ } \\
1550 \mathrm{~nm}\end{array}$ & $\mathrm{~dB}$ & 0.15 \\
\hline
\end{tabular}

\section{Result and Discussion}

The dual-wavelengths fiber laser provides the required sources for generating the converted signal based on FWM in the highly nonlinear optical in the ring cavity as shown in Fig. 4. The generation of this converted signal is depends on the power of the pump and signal wavelengths where a strong pump transfers its energy to create this converted signal as shown in Fig. 4. To achieve the highest converted signal output power, the pump power should be higher than the signal power and this can be done by controlling the cavity loss using variable optical coupler.
Hence, the highest output power of the converted signal with $-16.8 \mathrm{dBm}$ is obtained when the pump and signal powers are set to $+13.3 \mathrm{dBm}$ and $+5 \mathrm{dBm}$ respectively. From Fig. 4 , it can be infer that the converted signal power increases when the pump power is nearing the signal power. In addition, an additional idler wavelength is generated when the pump power is almost the same with the signal power. This is due to the power of the first idler is enough to generate the additional idler wavelength.

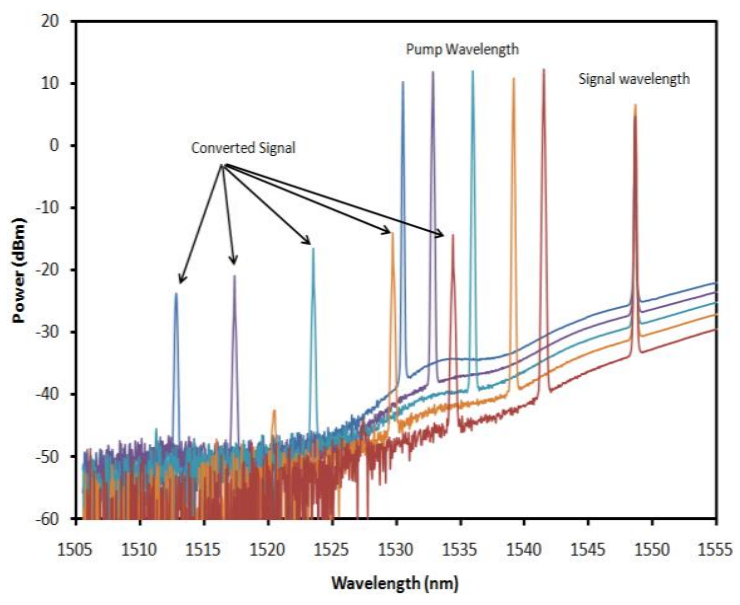

Fig. 4 Spectra of the FWM at different pump wavelength for different AWG channel.

In Fig. 5, the FWM conversion efficiency of dual-wavelength fiber laser as a function of wavelength detuning is plotted based on calculation and measurement values. Measurement of the conversion efficiency from the spectrum obtained using OSA is shown in Fig. 6, where the FWM conversion efficiency $\eta(\mathrm{dB})=P_{\text {signal }}(\mathrm{dBm})-P_{F W M}(\mathrm{dBm})$. The calculation of FWM conversion efficiency depends on the phase mismatch around the zero-dispersion wavelength and was calculated using equation (1). From equation (5), the FWM conversion efficiency depends on the fiber dispersion, wavelength detuning, fiber length and pump power [18]. Fig. 5 shows that the FWM conversion efficiency obtained experimentally rapidly decreases when the wavelength detuning exceeds $12 \mathrm{~nm}$ which is $3 \mathrm{~nm}$ shorter than the calculated value of $15 \mathrm{~nm}$. The possible reason for this discrepancy could be the larger phase mismatch experienced at larger wavelength separation [21]. Theoretically, the FWM conversion efficiency is higher when the pump wavelength is at 
zero-dispersion wavelength. Conversely, from the experiment carried out, the FWM conversion efficiency at zero-dispersion wavelength with the same wavelength detuning is lower compared to one at $1560 \mathrm{~nm}$ region due to the higher ASE level in this region as shown in Fig. 2. This is due to the emission from the $11 \mathrm{~m}$ EDF is shifted to a longer wavelength. In short, the use of the longer EDF contributes to a higher output power which will enhance the FWM effect. However, the maximum FWM conversion efficiency has shifted to a longer wavelength specifically the $1560 \mathrm{~nm}$ region. In this experiment, the work to measure the FWM conversion efficiency curve in the $1560 \mathrm{~nm}$ region is limited by the operating wavelength of AWG which covers the wavelength range from $1530.5 \mathrm{~nm}$ to $1548.6 \mathrm{~nm}$.

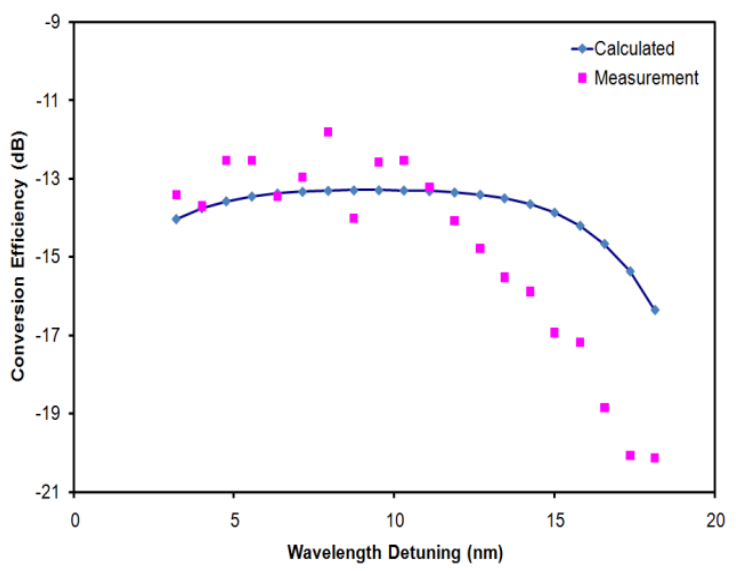

Fig. 5 FWM conversion efficiency versus wavelength detuning in HNLF for the dualwavelength fiber laser.

Based on equation (6), the FWM output power obtained from calculation is presented in Fig. 7. It shows that the FWM output power is around $-22 \mathrm{dBm}$ at wavelength detuning from $5 \mathrm{~nm}$ to $15 \mathrm{~nm}$ with the input pump and signal powers set at $+13.3 \mathrm{dBm}$ and $+5 \mathrm{dBm}$, respectively.

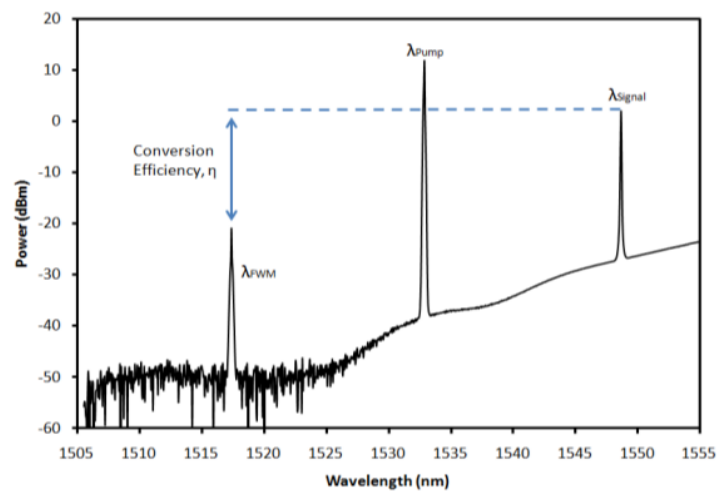

Fig. 6 Measurement of conversion efficiency in the OSA.

However, the FWM output power obtained from measurement varies from $16 \mathrm{dBm}$ to $-20 \mathrm{dBm}$ with wavelength detuning from $3 \mathrm{~nm}$ to $18 \mathrm{~nm}$, respectively. The output power slowly decreased when the wavelength detuning increased and this can be verified from calculation.

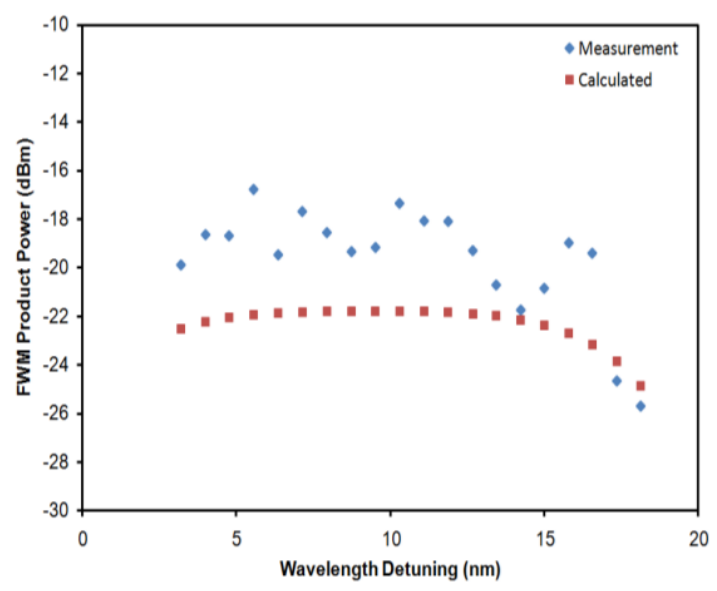

Fig. 7 FWM output power versus wavelength detuning between pump and signal.

Fig. 8 shows the variation of conversion efficiency of FWM with pump power, for a wavelength detuning of $8 \mathrm{~nm}$ in a high nonlinear fiber length of $100 \mathrm{~m}$. The conversion efficiency of FWM is proportional to the pump and signal power as can be explained based on equation (5). Since fiber laser power plays an important role in the generation of FWM, it is important to have high pump power to obtain the high conversion efficiency of FWM as shown in Fig. 8. A power of $21.4 \mathrm{~mW}(+13.3 \mathrm{dBm})$, for example, is needed to obtain the conversion efficiency of $-11 \mathrm{~dB}$. However, if the pump 
power less than $1 \mathrm{~mW}$, the conversion efficiency drops by about $19 \mathrm{~dB}$ compared to the maximum value.

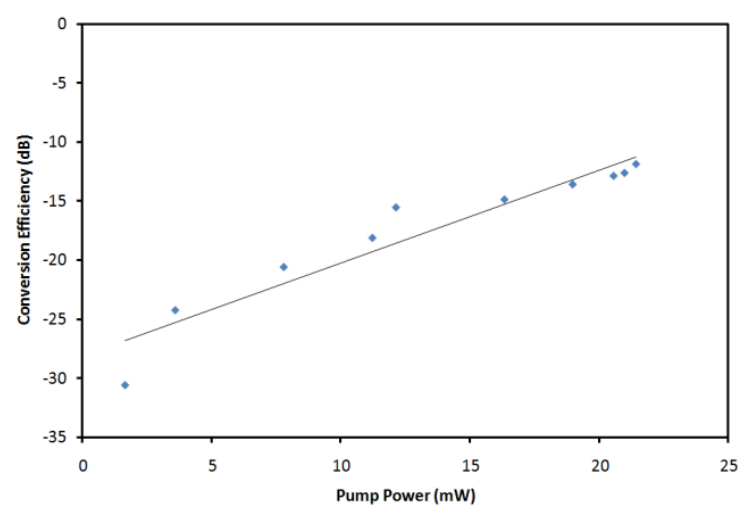

Fig. 8 FWM output power versus input pump power.

Fig. 9 shows the SNR of the converted signal against the wavelength detuning. Results show that the SNR varies between $30 \mathrm{~dB}$ to $40 \mathrm{~dB}$ with wavelength detuning up to $15 \mathrm{~nm}$. Fig. 9 also shows that closer wavelength detuning results in lower SNR because the peak of FWM product power overlaps with the ASE generated by the EDF. The characteristics of the FWM in dualwavelength fiber laser of HNLF are important for wavelength converter application to avoid distortion of the output signal.

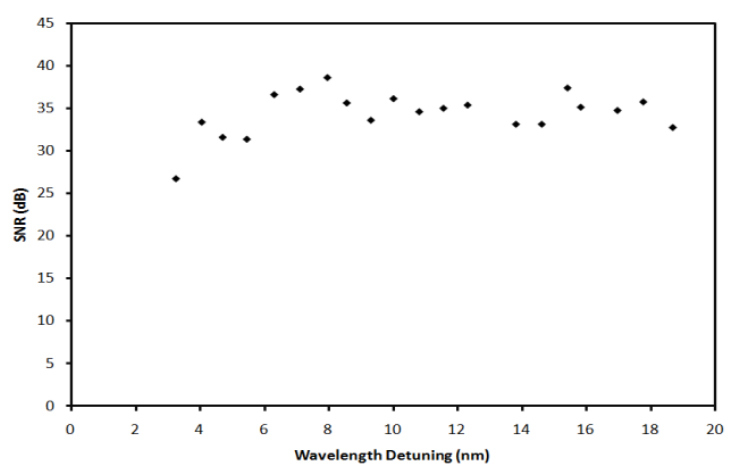

Fig. 9 Optical signal to noise ratio against

\section{Conclusion} wavelength detuning.

In this paper, we demonstrate the use of a selfconstructed dual wavelength fiber laser tunable in the $1550 \mathrm{~nm}$ region as pump source and signal probe for FWM generation in a highly non-linear fiber. The tunability is achieved using an arrayed waveguide grating which provides different wavelength combinations of the dual-wavelength fiber laser output and can be tuned from $1530.47 \mathrm{~nm}$ to $1548.61 \mathrm{~nm}$ with a spacing of $100 \mathrm{GHz}$. The highly nonlinear fiber has a nonlinear coefficient, $\gamma$ of $10.8 \mathrm{~W}^{-1} \mathrm{~km}^{-1}$. To achieve higher FWM output power the pump power of the dual wavelength fiber laser has to be higher than that of the signal probe. The optimum measured values of pump and signal power are $+13.3 \mathrm{dBm}$ and $+5 \mathrm{dBm}$ respectively, limited buy the current experimental setup. In our case, the FWM conversion efficiency is higher when the pump and signal wavelength are set at the highest ASE level instead of the zero-dispersion wavelength. This means that the FWM conversion efficiency is more dependent on the wavelength detuning between pump and signal wavelength than the zero-dispersion wavelength in a dualwavelength fiber laser configuration.

\section{References}

[1] Danielsen, S.L., Hansen, P.B. \& Stubkjaer, K.E. (1998). "Wavelength Conversion in Optical Packet Switching" in Journal of Lightwave Technology, Vol. 16. No. 12 pp. 2095.

[2] Kovacevic, M. \& Acampora, A. (1996). "Benefits of Wavelength Translation in All-Optical ClearChannel Networks" in Journal on Selected Areas in Communications, Vol. 14. No. 5 pp. 868-880.

[3] Yoo, S.B. (1996). "Wavelength Conversion Technologies for WDM Network Applications" in Journal of Lightwave Technology, Vol. 14. No. 6 pp. 955-966.

[4] Patrick, D.M. \& Manning, R.J. (1994). "20 Gbit/S Wavelength Conversion using Semiconductor Nonlinearity," in Electronics Letters, Vol. 30. No. 3 pp. 252-253.

[5] Durhuus, T., Joergensen, C., Mikkelsen, B., Pedersen, R.J.S. \& Stubkjaer, K.E. (1994). "All Optical Wavelength Conversion by SOA's in a Mach-Zehnder Configuration," in Photonics Technology Letters, Vol. 6. No. 1 pp. 53-55.

[6] Pan, X. \& Koch, T.L. (1995). "Intensity Noise Characteristics of a Mach-Zehnder Wavelength 
Converter," in Photonics Technology Letters, Vol. 7. No. 11 pp. 1276-1278.

Inoue, K. \& Oda, K. (1995). "Noise Suppression in Wavelength Conversion using a Light-Injected Laser Diode," in Photonics Technology Letters, Vol. 7. No. 5 pp. 500-501.

[8] Kikuchi, N. \& Sasaki, S. (1993). "Noise Analysis for Optical Frequency Conversion using Nearly Degenerate Four Wave Mixing in Semiconductor Optical Amplifier" in Journal of Lightwave Technology, Vol. 11. No. 56 pp. 819-828.

[9] Schunk, N., Großkopt, G., Ludwig, R., Schnabel, R. \& Weber, H.G. (1990). "Frequency Conversion by NearlyDegenerate Four-Wave Mixing in Traveling-Wave Semiconductor Laser Amplifiers," in Proceedings J (Optoelectronics), Vol. 137. No. 4 pp. 209-214.

[10] Kothari, N.C. \& Blumenthal, D.J. (1996). "Influence of Gain Saturation, Gain Asymmetry, and Pump/Probe Depletion on Wavelength Conversion Efficiency of FWM in Semiconductor Optical Amplifiers" in Journal of Quantum Electronics, Vol. 32. No. 10 pp. 1810-1816.

[11] D'Ottavi, A., Iannone, A., Mecozzi, A., Scotti, S., Spano, P., Dall'Ara, R. \& Guekos, G. (1995). "Efficiency and Noise Performance of Wavelength Converters Based on FWM in Semiconductor Optical Amplifiers" in Photonics Technology Letters, Vol. 7. No. 4 pp. 357-359.

[12] Chow, K.K., Mak, M.W.K., Shu, C., \& Tsang, H.K. (2002). "Widely Tunable All-Optical Wavelength Converter using a Fiber Ring Cavity Incorporating a Semiconductor Optical Amplifier" in Optics Communications, Vol. 203, No.1 pp. 101-106.

[13] Chow, K.K., Shu, C., Mak, M.W.K. \& Tsang, H.K. (2002). "Widely Tunable Wavelength Converter using a Double-Ring Fiber Laser with a Semiconductor Optical Amplifier" in Photonics Technology Letters, Vol. 14. No. 10 pp. $1445-1447$.
[14] Agrawal, G.P. (1995). Nonlinear Fiber Optics. $2^{\text {nd }}$ Edition, Academic Press, pp. 322-324.

[15] Hill, K.O., Johnson, D.C., Kawasaki, B.S. \& Macdonald, R. I. (1978). “CW Three-Wave Mixing in Single-Mode Optical Fibers," in Journal of Applied Physics, Vol. 49. No. 10 pp. 50985106.

[16] Shibata, N., Braun, R. P. \& Waarts, R. G. (1987). "Phase-Mismatch Dependence of Efficiency of Wave Generation Through Four-Wave Mixing in a Single-Mode Optical Fiber," in Journal of Quantum Electronics, Vol. 23. No. 7 pp. 12051210.

[17] Deepa, R. \& Vijaya, R. (2007). "Generalised Dispersive Phase and its Effect on Four Wave Mixing in Fibers," in Optics Communications, Vol. 269. No. 1 pp. 206-214.

[18] Song, S., Allen, C. T., Demarest, K. R. \& Hui, R. (1999). "IntensityDependent Phase-Matching Effects on Four-Wave Mixing in Optical Fibers," in Journal of Lightwave Technology, Vol. 17. No. 11 pp. 22852290.

[19] Inoue, K. (1992). "Four-Wave Mixing in an Optical Fiber in the ZeroDispersion Wavelength Region" in Journal of Lightwave Technology, Vol. 10. No. 11 pp. 15531561.

[20] Hasegawa, T., Nagashima, T. \& Sugimoto, N. (2008). "Determination of Nonlinear Coefficient and GroupVelocity-Dispersion of Bismuth-Based High Nonlinear Optical Fiber by FourWave-Mixing," in Optics Communications, Vol. 281. No. 4 pp. 782-787.

[21] Chen, L.R. \& Gu, X. (2007). "DualWavelength Yb-Doped Fiber Laser Stabilized Through Four-Wave Mixing," in Optics Express, Vol. 15. No. 8 pp. 5083-5088. 